\title{
A Frame Size Adjustment with Sub-Frame Observation for Dynamic Framed Slotted Aloha
}

\author{
Robithoh Annur \\ Department of Computer \\ and Communications Technology \\ Universiti Tunku Abdul Rahman \\ Perak, Malaysia
}

\author{
Suvit Nakpeerayuth \\ and Lunchakorn Wuttitsittikulkij \\ Department of Electrical Engineering \\ Chulalongkorn University \\ Bangkok, Thailand
}

\begin{abstract}
In this paper, a simple frame size adjustment of dynamic framed slotted Aloha for tag identification in RFID networks is proposed. In dynamic framed slotted Aloha, the reader is required to announce the frame size for every frame. To achieve maximum system efficiency, it is essential to set the frame size according to the number unidentified tags appropriately. The proposed approach utilizes the information from a portion of the frame to adjust the size of the next frame. Simulation results show that the smaller number of observed slots results in faster frame adjustment and higher throughput. Compared to the existing anti-collision algorithms, the proposed approach achieves higher throughput and higher identification rate.
\end{abstract}

Keywords-anti-collision tag identification; RFID; Framed slotted Aloha; frame adjustment

\section{INTRODUCTION}

Radio Frequency Identification (RFID) is a short range wireless communication technology for identifying a specific object. It is also known as electronic tag. Unlike barcode, it does not require line-of-sight. Moreover, it is able to read, transmit and store the information of the objects. With the development of the RFID technology, it is widely applied in many sectors such as retail trade, services, manufacturing, supply chain management, logistics, information industry, and medical applications. A simple RFID system consists of tags with data memory and antenna, interrogators/ readers, and host with data processing software. A problem so called tag collision may occur when at least two tags respond to the same reader simultaneously. An anti-collision algorithm is performed in such a way to solve this problem and hence increases the tag reading efficiency. To achieve fast tag identification, many tag anti-collision protocols have been proposed. They can be broadly classified into two categories: tree-based and Aloha-based.

Tree based RFID tag anti-collision protocols such as Query tree, M-ary query tree, collision tree [1], consecutive collision bit mapping algorithm (CCMA)[2], Dual Prefix Probe Scheme (DPPS) [3] are developed from the traditional tree algorithm by Capetanakis [4] where the colliding tags are recursively split into subgroups based on the position of the bits in their IDs. However, distribution of tags' ID and collision bit position are the major factors affecting the tag identification speed.

This paper focuses on Aloha based anti-collision algorithm. In the application of RFID systems, framed slotted Aloha receives much attention compared to other types of Aloha based algorithms. To cope with the problem of tag starvation, many researches have applied adaptive frame size, so called dynamic framed slotted Aloha [5].

In dynamic framed slotted Aloha, the frame size is set dynamically equal to the number of remaining unidentified tags. In fact, the reader usually does not have any information regarding the number of tags. This leads to necessity of an estimation of the number of tags in each frame $[6,7]$.

In literature, many tag estimate methods have been addressed by observing all slots in the previous frame to find the number of idle $(I)$, successful $(S)$ and collision $(C)$. Schoute's method suggests that the tag number estimate is $\hat{n}=2.39 C$. Vogts method utilizes $I, S$ and $C$ in the tag estimation using mean square error. By assuming that tags in each frame have a multinomial distributition, Chen [8] and Vahedi [9] present a more accurate tag number estimate using a posteriori probability distribution, but at a cost of increased complexity [10]. To achieve a good estimate of number of tags with much reduced complexity, [10] proposed an Improved Linearized Combinatorial Model (ILCM) which uses an efficient interpolation method that is easy to be implemented.

Different from the aforementioned estimate methods that observe the entire slots in the frame, some existing frame size adjustment methods perform frame breaking policy whereby only a portion of the frame is observed. This method allows the reader to end the current frame and start a new frame when an inappropriate frame size is detected. An example of this methode is $Q$ algorithm that is adopted in the EPC class 1 Gen 2 of RFID standard [11]. This algorithm is an effective approach to change the frame size of dynamic framed slotted Aloha without requiring the knowledge of the number of unidentified tags, i.e. no explicit estimation of tag numbers is required at the end of each frame. The $Q$ algorithm basically adjusts a parameter called $Q$ with a parameter $c$ in response to the outcome of tag transmission on a slot by slot basis. The $Q$ algorithm is simple, but it is not fully clear how the parameter $c$ should be set $[10,12]$, despite some efforts by $[13,14]$. Chen in [12] (W-T Chen's algorithm) proposed an tag estimation method namely a feasible and easy to implement anti-collision algorithm. When it detects that the frame size is not in the range of optimum value, the frame will end and continue the identification process with new frame. This $\mathrm{W}-\mathrm{T}$ Chen's algorithm provides a good tag estimation. However, it requires non-empty slot in the first slot of the frame that may not always be true. 


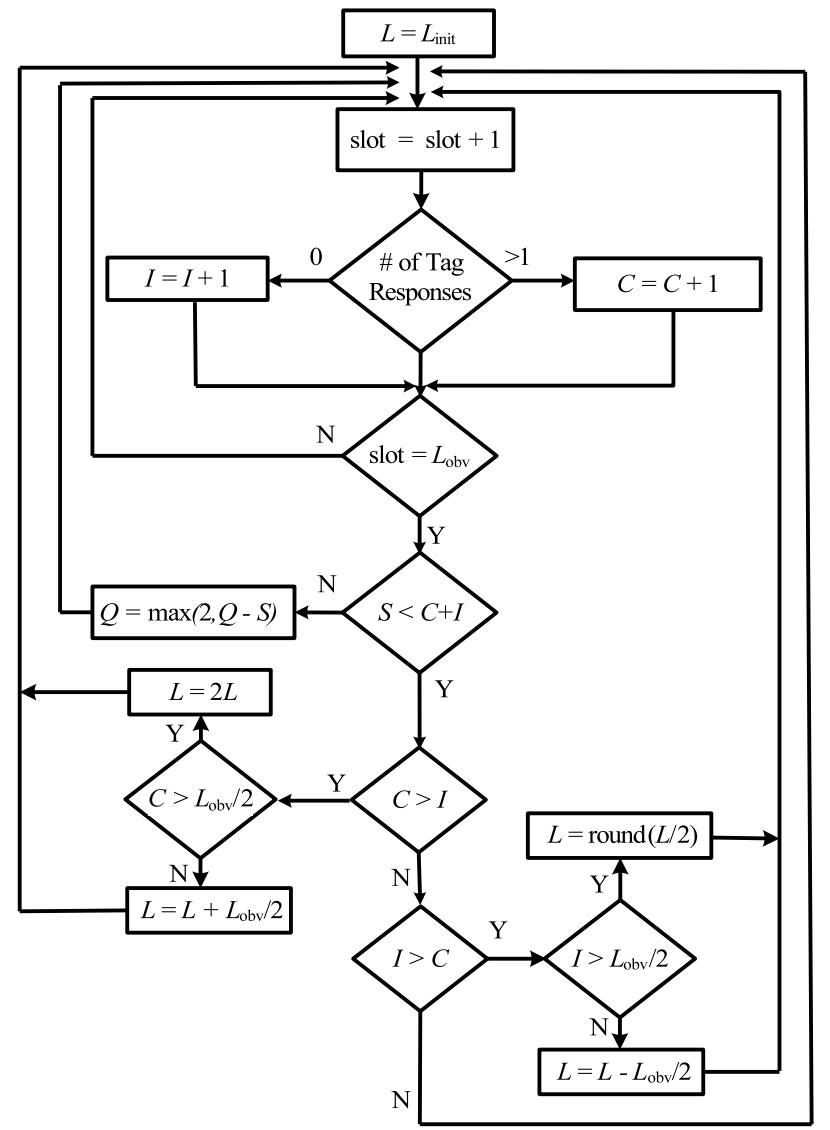

Fig. 1. The flow diagram of the proposed algorithms

In this letter, a simple frame breaking policy for dynamic framed slotted Aloha frame size adjustment is proposed. This aims to reduce the computational complexity and improve the identification speed of the system. The propose algorithm relies on the ratio between the number of collisions and idle slots in a portion of the frame. The proposed method does not apply complex computation but can achieve a considerable performance.

\section{System Model and Frame AduUstment DESCRIPTION}

The anti-collision protocol used in this paper is based on the dynamic framed slotted Aloha. To maximize the success probability in each frame, the frame size should be set properly according to the number of collided tags. There will be many collision or idle slots if the frame size is too small or too large compared the number of collided tags.

It is well known that the ideal DFSA achieves a maximum throughput of $36.8 \%$ when $L=N$. For this ideal case, the system required the number of collided tags in each frame to set the frame size that can be done by performing tag estimation. Usually, to estimate the number of tags in a frame, it is required to observed all slots in the frame. This approach may not always provide an effective result when the frame size is inappropriate due to error in the estimation. In this case, the reader can break the current frame and initiates a frame with different size.
The proposed frame size adjustment with a breaking policy that is based on the ratio between empty and collision slots in the observed slots in the frame. Fig. 1 depicts the proposed frame size adjustment for framed slotted Aloha. At the beginning of the identification process, the reader will broadcast the initial frame size, denoted as $L_{\text {init }}$ and the observed slots denoted as $L_{o b v}$ that is the portion of the frame (first several slots in the frame). In every frame, the reader will initiated a new frame after observing $L_{o b v}$. The frame size may be shrinked or enlarged depending on the ratio between empty and collision slots in the observation. If the number of collision slots is greater than the number of idle slots, it is expected that the frame size is too small compared to the number of collided tags.For this case, it is further checked whether the number of collisions is greater than the Lobv/2. If so, the frame size will increase by $L o b v / 2$, otherwise it will be doubled. On the other hand, if the number of collision slots is smaller than the number of idle slots, the frame size will reduce by $L o b v / 2$ if the number of idle slots is greater than Lobv/2 or will reduce by half for other cases. If the numbers of collision and idle slots are equal, the frame size remains unchanged.

\section{Simulation Results and Discussion}

This section presents the evaluation of the effectiveness of the proposed frame adjustment approach in terms of two different performance matrices namely throughput or system efficiency and read rate or identification rate.

It will first show the effect of different initial frame size, $L_{\text {init }}$ and $L_{o b v}$ on the throughput or system efficiency of the proposed algorithm. Computer simulations were conducted with the initial number of tags $(N)$ set up to 1000 tags and run the simulator for each scenario for 10,000 times. To show the effectiveness of the algorithms in resolving collisions, the results are presented in terms of throughput or system efficiency which is normally calculated as the ratio between the number success slots and the total number of slots spent in a collision resolution process that is given by:

$$
S E=\frac{S}{S+I+C}
$$

Fig. 2 shows the effect of different parameter settings of $L_{i n i t}$ and $L_{o b v}$ to the system efficiency. Three different settings of $L_{\text {init }}$ are considered, namely 16,32 , and 64 . It can be seen that the setting of $L_{\text {init }}$ have significant impact to the system efficiency. When the number of tags is far $L_{\text {init }}$, the system efficiency is considerably low, especially when the number of tags is less than the $L_{\text {init }}$. For example, for $L_{i n i t}=32$ and 64, the system efficiency drop to below 0.3 i.e. 0.22 and 0.125 , respectively, when the number of tags is 10 . Especially for case when $L_{\text {init }}=16$, the system efficiency is consistently above 0.345 for either small or large number of tags. From these results, $L_{\text {init }}=16$ can be considered as a proposed parameter setting.

To show the influence of the setting of $L_{o b v}$, four different portions of frame to be observed is considered; $L, L / 2, L / 4$, and $L / 8$. From Fig.2, it reveals that the setting of $L_{o b v}$ gives significant effect to the throughput. For the three different values of $L_{i n i t}$, each of these four values of $L_{o b v}$ presents the same fashion where the smaller number of observed slots, the 

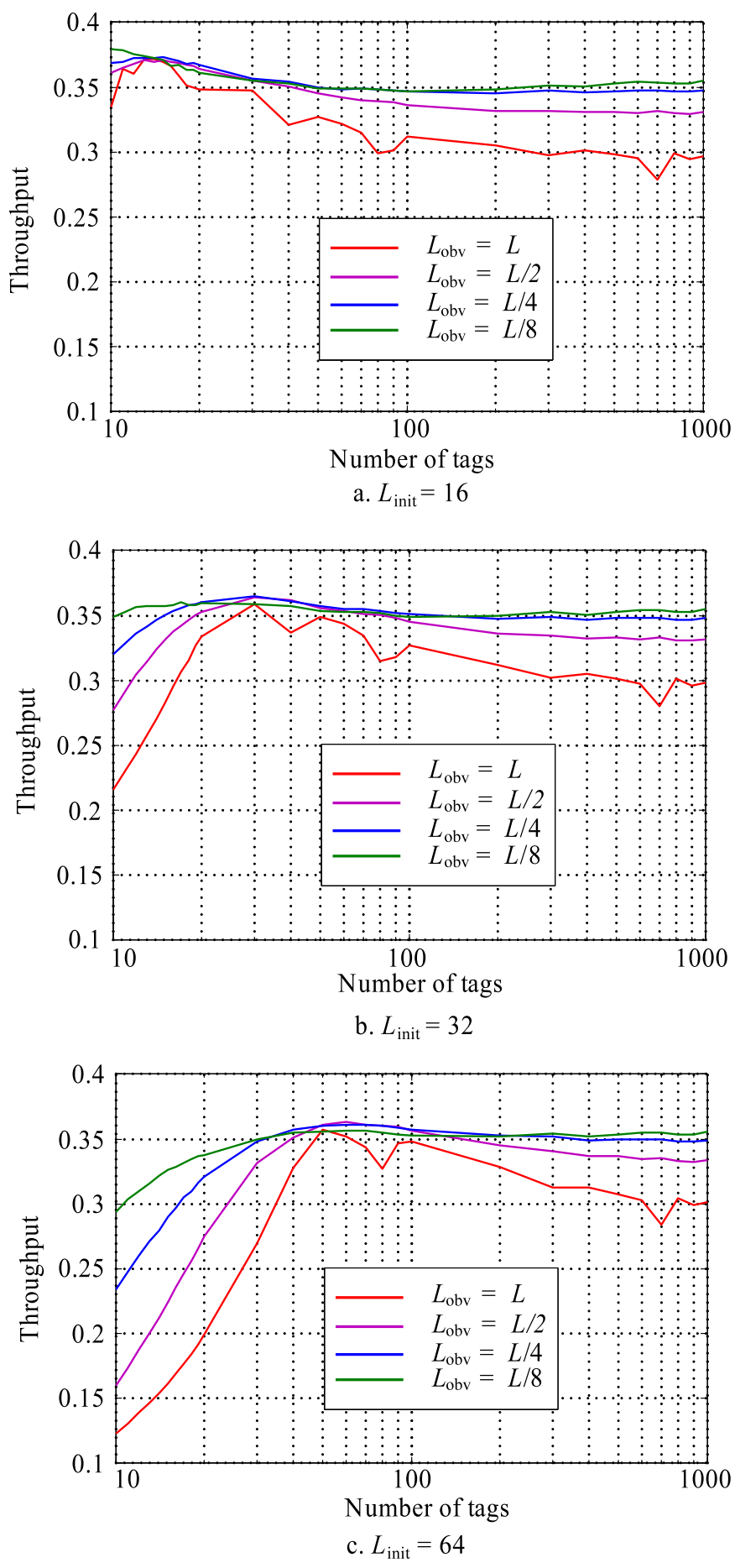

Fig. 2. Normalized throughput of the proposed approach under different initial value of $\mathrm{L}$

better performance is achieved. This is expected because when the observation is for the entire frame, the frame adjustment will be slow to reach the appropriate frame size for the number of remaining unidentified tags. Hence, there is high probability that the slots in the frame will be idle or collision. These reasons will create the drop in the system efficiency of the proposed approach. On the other hand, when small portion of frame is observed, the frame adjustment will be fast to

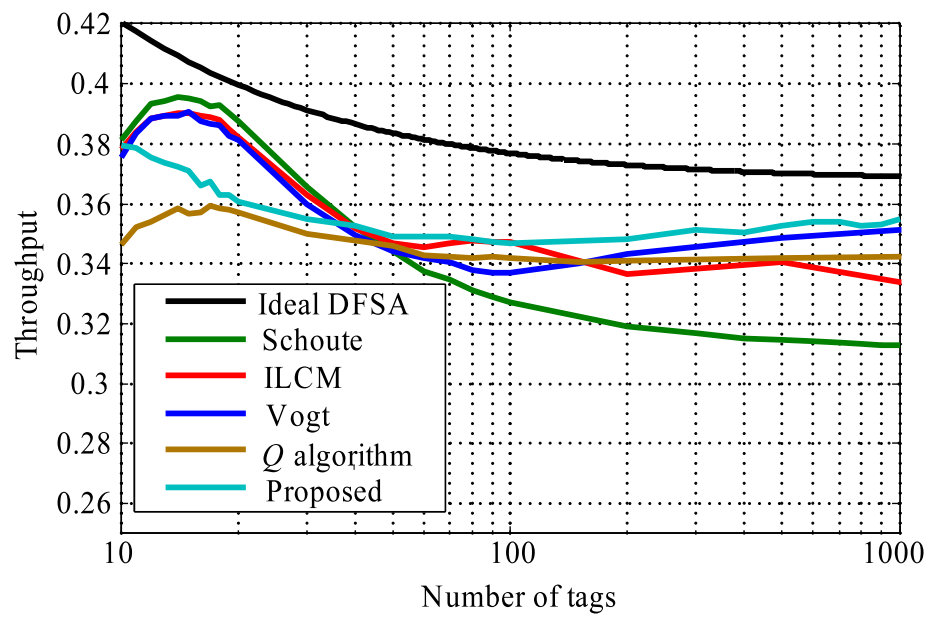

Fig. 3. Normalized throughput comparison between Aloha-based anticollision algorithms

reach the appropriate frame size. As a result, the probability of success can be maximized. From these results, it suggests that smaller $L_{o b v}$ is preferred to achieve a good performance for board range of number of tags.

Fig. 3 compares the throughput of the proposed approach with the existing anti-collision algorithms such as: ideal DFSA, Vogt, Schoutes, ILCM, and Q algorithm. For this comparison, the $L_{\text {init }}$ is set to 16 for all algorithms and $L_{o b v}$ is $L / 8$. It shows that Schoutes, Vogts, ILCM algorithms perform very well for small number of tags, i.e. above 0.36 efficiency for $N<30$. For larger $N$, these three existing algorithms exhibit different behaviors. The throughput of Schoutes algorithm drops relatively fast for larger values of $N$ (this is also suggested in [12]), e.g. for $N>200$ where the efficiency falls below 0.32 . The drop also happens to ILCM, but it still maintain the system efficiency of almost 0.34 at large $N$, i.e. $N>200$. For Vogts algorithm, the throughput will decrease up to below 0.34 at around $\mathrm{N}=80$ and will rise again up to above 0.34 for $N=1000$. For the well known $Q$ algorithm, the throughput is less than 0.36 and stable for 0.342 for large vale of $N$. The proposed algorithms can perform better than all other algorithms in most cases where it can achieve the system performance of almost $35 \%$ for the entire range of the number of tags.

Besides the throughput, this section will also compare the identification rate of the proposed approach and the existing anti-collision algorithms. Identification rate is defined as the total number of tags that is successfully identified in a given unit of time. It is referred here as tags/ second. To measure the identification rate, the timing in EPCglobal Class 1 Gen 2 RFID standard is referred. Duration for the empty, success and collisions are defined differently. This is because every slot condition reflects different step of communication between the tags and the reader as illustrated in Fig. 4. It can be seen that the duration of the empty, success and collisions can respectively be given by:

$$
T_{\text {idle }}=T_{\text {Qrep }}+T_{1}+T_{3}
$$

$T_{\text {success }}=T_{Q r e p}+T_{1}+T_{R N 16}+T_{2}+T_{A C K}+T_{1}+T_{E P C}+T_{2}$ 


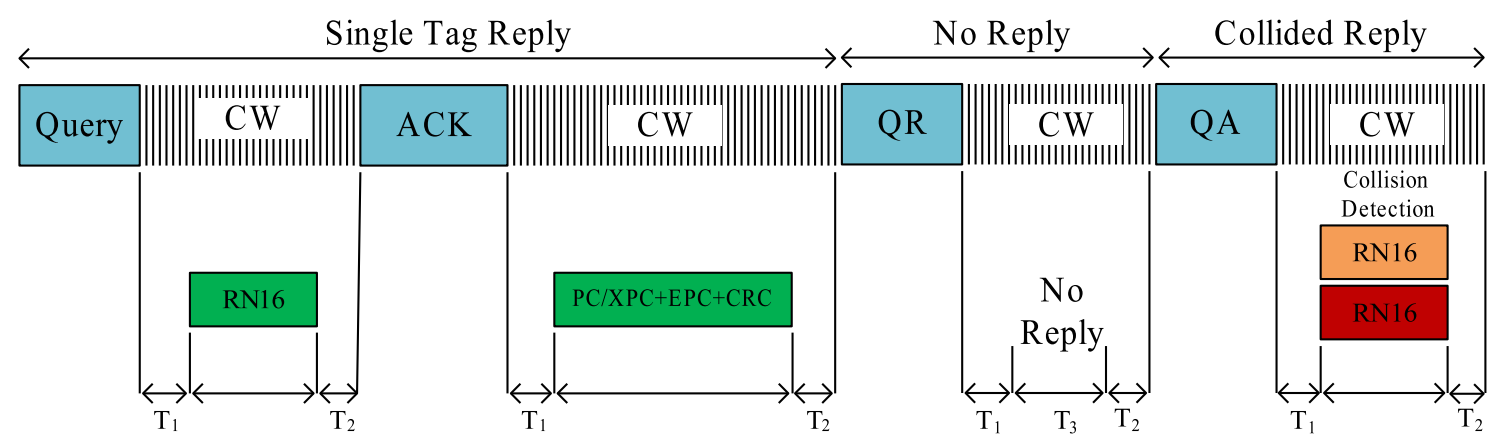

$\mathrm{QR}=$ Query Reply $\mathrm{QA}=$ Query Adjust $\mathrm{CW}=$ Continuous Wave

Fig. 4. Timing in EPCglobal Class 1 Gen 2

TABLE I. GEN2 PARAMETER

\begin{tabular}{|l|l|}
\hline Parameter & Value \\
\hline Tari & $6.25 \mu \mathrm{s}$ \\
\hline TRCal & $33.33 \mu \mathrm{s}$ \\
\hline T1 & $33.33 \mu \mathrm{s}$ \\
\hline T2 & $15.63 \mu \mathrm{s}$ \\
\hline T3 & $0.516 \mu \mathrm{s}$ \\
\hline TFS & $35.94 \mu \mathrm{s}$ \\
\hline TQuery & $414.06 \mu \mathrm{s}$ \\
\hline TQueryRep & $104.69 \mu \mathrm{s}$ \\
\hline TACK & $345.31 \mu \mathrm{s}$ \\
\hline TRN16 & $337.50 \mu \mathrm{s}$ \\
\hline TEPC & $1.7 \mathrm{~ms}$ \\
\hline
\end{tabular}

$$
T_{\text {colision }}=T_{Q \text { rep }}+T_{1}+T_{R N 16}+T_{2}
$$

The identification rate can be then calculated by:

$$
\text { rate }=\frac{S}{T_{\text {success }} S+T_{\mathrm{idle}} I+T_{\text {colision }} C}
$$

The EPCglobal Class 1 Gen 2 RFID standard can operate in several different tag- reader link rates. It uses tag- reader link rates of $640 \mathrm{kbps}$ with the detail parameters given in Table. I. It can be seen that the proposed frame adjustment approach offer high identification rate for larger range of the number of tags.

\section{CONCLUSION}

Frame size selection is a major challenge in dynamic framed slotted Aloha based RFID anti-collision algorithm since the reader does not have any information regarding the number of tags in the inventory process. This paper has presented a simple frame size adjustment of dynamic framed slotted Aloha that allows early frame adjustment. The algorithm will only observe first few slots of the frame and initiate a new frame. Based on the comparison between the number of idle slots and collisions in the number of observed slots, the new frame size is determined. The results show that this simple approach offers higher throughput and identification rate compared to the other existing algorithms.

\section{ACKNOWLEDGMENT}

The authors would like to thank to Ratchadapiseksomphot Endowment Fund from Chulalongkorn University

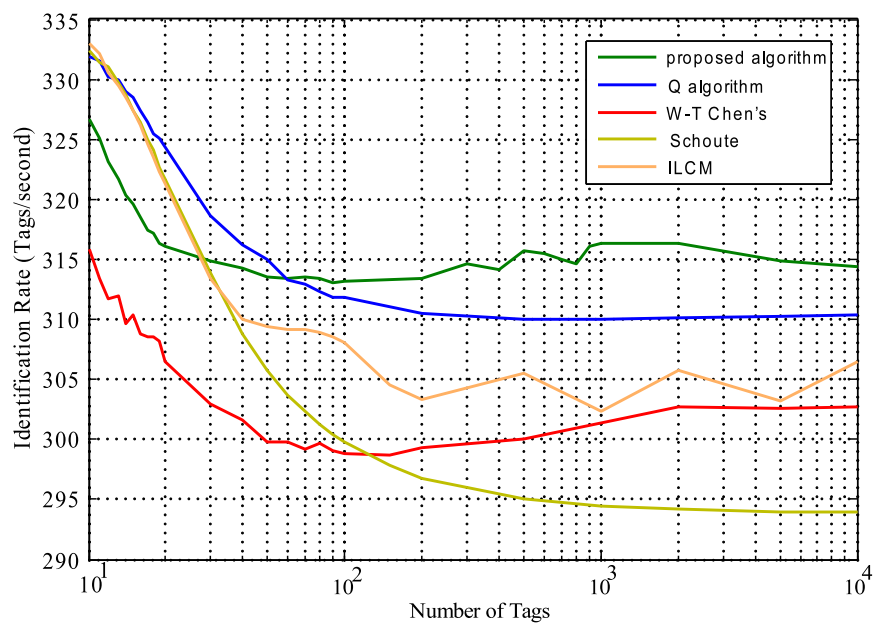

Fig. 5. Identification rate comparison between Aloha-based anti-collision algorithms

and AUN/SEED-Net program, and UTARRF (IPSR/RMC/UTARRF/2015-C2/R02) from Universiti Tunku Abdul Rahman for the financial support.

\section{REFERENCES}

[1] X. Jia, Q. Feng, and L. Yu, "Stability analysis of an efficient anti-collision protocol for rfid tag identification," IEEE Transactions on Communications, vol. 60, no. 8, pp. 2285-2294, August 2012.

[2] H. J.-1. SU Jian, WEN Guang-jun, "An efficient rfid anticollision algorithm for iso 18000-6b protocol," Chinese Journal of Electronics, vol. 42, no. 12, p. 2515, 2014.

[3] J. Su, Z. Sheng, G. Wen, and V. C. M. Leung, "A time efficient tag identification algorithm using dual prefix probe scheme (DPPS)," IEEE Signal Processing Letters, vol. 23, no. 3, pp. 386-389, March 2016.

[4] J. Capetanakis, "Tree algorithms for packet broadcast channels," Information Theory, IEEE Transactions on, vol. 25, no. 5, pp. 505-515, Sep 1979.

[5] F. Schoute, "Dynamic frame length aloha," Communications, IEEE Transactions on, vol. 31, no. 4, pp. 565-568, Apr 1983.

[6] H. Vogt, "Efficient object identification with passive RFID tags," in Proceedings of the First International Conference on Pervasive Computing, ser. Pervasive '02. London, UK: Springer-Verlag, 2002, pp. 98-113.

[7] C. Floerkemeier, "Bayesian transmission strategy for framed 
Aloha based RFID protocols," in RFID, 2007. IEEE International Conference on, March 2007, pp. 228-235.

[8] W.-T. Chen, "An accurate tag estimate method for improving the performance of an RFID anticollision algorithm based on dynamic frame length Aloha," Automation Science and Engineering, IEEE Transactions on, vol. 6, no. 1, pp. 9-15, Jan 2009.

[9] E. Vahedi, V. Wong, I. Blake, and R. Ward, "Probabilistic analysis and correction of Chen's tag estimate method," Automation Science and Engineering, IEEE Transactions on, vol. 8, no. 3, pp. 659-663, July 2011

[10] P. Solic, J. Radic, and N. Rozic, "Energy efficient tag estimation method for Aloha-based RFID systems," Sensors Journal, IEEE, vol. 14, no. 10, pp. 3637-3647, Oct 2014.

[11] "EPCglobal, EPC radio-frequency identity protocols class-1 generation-2 UHF RFID protocol for communications at 860 MHz-960 MHz," no. 1.2.0, 2008.

[12] W.-T. Chen, "A feasible and easy-to-implement anticollision algorithm for the EPCglobal UHF Class-1 Generation-2 RFID protocol," Automation Science and Engineering, IEEE Transactions on, vol. 11, no. 2, pp. 485-491, April 2014.

[13] D. Lee, K. Kim, and W. Lee, " $Q+$-algorithm: An enhanced RFID tag collision arbitration algorithm," in Prooceeding Conference Ubiquitous Intelligence Comput, 2007, pp. 23-32.

[14] H. Wu, Y. Zeng, J. Feng, and Y. Gu, "Binary tree slotted aloha for passive RFID tag anticollision," Parallel and Distributed Systems, IEEE Transactions on, vol. 24, no. 1, pp. 19-31, Jan 2013. 